\title{
A generalization of Reiner's mathematical model for wet sand
}

\author{
Mehrdad Massoudi* \\ U.S. Department of Energy, National Energy Technology Laboratory (NETL), 626 Cochrans Mill Road, P.O. Box 10940, Pittsburgh, PA 15236, USA
}

\section{A R T I C L E I N F O}

\section{Article history:}

Received 24 November 2010

Received in revised form 18 April 2011

Available online 14 May 2011

Dedicated to the memory of Dr. Alec Stewart, my freshman (1976) physics professor at the University of Pittsburgh and the (Founding) Dean of University Honors College.

\section{Keywords:}

Continuum mechanics

Wet sand

Dilatancy

Shear flow

Non-Newtonian fluids

Granular materials

\begin{abstract}
A B S T R A C T
In this paper we modify the constitutive relation derived by Reiner (1945), to describe dilatancy in wet sand, by suggesting that the shear viscosity would depend on the shear rate and the volume fraction. We then look at the flow of a saturated densely packed bed of particles (with liquid in the pores) between two horizontal flat plates. We obtain exact solutions for a very special case.
\end{abstract}

Published by Elsevier Ltd.

\section{Introduction}

Reynolds (1885) observed that for a shearing motion to occur in a bed of closely packed particles, the bed must expand to increase the volume of its voids. He termed this phenomena "dilatancy". Reynolds (1886) used this idea to describe the capillary action in wet sand. The concept of dilatancy is generally taken to be the expansion of the voidage that occurs in a tightly packed granular arrangement when it is subjected to a deformation (shear). That is, in order for a shearing motion to occur in a bed of closely packed spheres, the bed must expand by increasing its void volume. The work of Reynolds was followed by the experimental studies of Jenkin (1931), Rowe (1962), Andrade and Fox (1949), and Bolton (1986) to name a few (see Massoudi and Mehrabadi, 2001 for details). In fact, Reiner $(1945,1948,1958)$ was one of the first who derived a non-Newtonian fluid model to predict dilatancy in wet sand. Even though this model does not take into account how the voidage (volume fraction) affects the stress, Reiner was able to show that application of a non-zero shear stress produces a change in volume.

Reiner's paper (1945) has had a major impact on the developments of constitutive modeling of non-linear fluids. In fact, as

\footnotetext{
* Tel.: +1 4123864975 .

E-mail addresses: Massoudi@netl.doe.gov, mehrdad.massoudi@netl.doe.gov
}

Truesdell (1965, p. 1) in his introduction to Reiner's paper, which is part of an edited collection of important articles on rational mechanics, says: "The paper here reprinted begins the rebirth of continuum mechanics as a rational science. In spirit it is transitional between the older work and the new... While the fraction of this paper containing results that are both correct and new is a small one, it is that fraction that matters. . While none of its results or proofs can now be accepted, this paper has had influence, direct and indirect, on particularly all subsequent work in rational continuum mechanics." It is in this spirit that we will try to generalize, or in a sense improve, Reiner's model, by making its shear viscosity dependent not only on the shear rate but also on the volume fraction. In fact, it needs to be mentioned that as far as the rheological issues are concerned, it is widely accepted that the Reiner-Rivlin fluid has been replaced by the Rivlin-Ericksen (1955) fluids of grade two.

In general, the behavior of granular materials (wet or dry) is governed by interparticle cohesion, friction, collisions, etc. Granular materials present a special challenge to engineers and scientists. It is a multi-disciplinary field, and one can study it from many different perspectives (see the books by Mehta, 1994; Duran, 2000; Antony et al., 2004). From a theoretical perspective, there are fundamentally two different and distinct, yet related methods that can be used: one is the statistical theories and the other the continuum theories.

In the statistical theories, either a particle dynamics approach (simulation) or modified forms of the kinetic theory of gases are 
used. These two techniques, especially with the advent of faster and more efficient computers have become very popular in the last two decades. There are many review articles which deal with the statistical theories (Hermann, 1999; Hermann and Luding, 1998) and kinetic theories as applied to granular materials (Massoudi and Boyle, 2001; Goldhirsch, 2003). In the continuum approach one assumes that the material properties of the ensemble may be represented by continuous functions so that the medium may be divided infinitely without losing any of its defining properties (Massoudi, 2004). There are also studies which focus on the onset of the flow, or the yield condition, and therefore techniques of theory of plasticity have been used. Theories of structured continua have also been used (Ahmadi, 1980) and there have been other attempts where the stress tensor is assumed to be composed of two parts: a rate-independent part, similar to a yield condition, for the frictional flow regime, and a rate-dependent part for the viscous flow regime (Tardos, 1997).

In this paper we modify and generalize the constitutive relation derived by Reiner (1945) to describe dilatancy in wet sand by suggesting that the shear viscosity would depend on the shear rate and the volume fraction. We then study the flow of a densely packed bed of particles (with liquid in the pores) between two horizontal flat plates. We show that it is possible to obtain exact solutions for a very special case. In Section 2, we present the governing equations, and in Section 3, a brief discussion of the constitutive relation for the stress tensor $\mathbf{T}$ is provided. In Section 4 the equations of motion for the shearing flow between two flat plates along with the exact solution are presented.

\section{Governing equations}

The balance laws, in the absence of thermo-chemical and electromagnetic effects, are the conservation of mass, linear momentum, and angular momentum (Truesdell and Noll, 1992). The conservation of mass in the Eulerian form is given by:

$$
\frac{\partial \rho}{\partial t}+\operatorname{div}(\rho \mathbf{u})=0
$$

where $\partial / \partial t$ is the partial derivative with respect to time. The balance of linear momentum is

$\rho \frac{d \mathbf{u}}{d t}=\operatorname{div} \mathbf{T}+\rho \mathbf{b}$

where $d / d t$ is the total time derivative, given by $(d(\cdot) / d t)=(\partial(\cdot) / \partial t)+[\operatorname{grad}(\cdot)] \mathbf{u}, \mathbf{b}$ is the body force, and $\mathbf{T}$ is the Cauchy stress tensor. The balance of angular momentum (in the absence of couple stresses) yields the result that the Cauchy stress is symmetric. For a complete study of a thermo-mechanical problem, the Second Law of Thermodynamics has to be considered. Since there is no general agreement on the functional form of the constitutive relation and since the Helmholtz free energy is not known, a complete thermodynamical treatment of the present model used in our studies is lacking. In order to 'close' the governing equations, we need constitutive relations for $\mathbf{T}$.

\section{Constitutive equations}

Most granular materials exhibit two unusual and peculiar characteristics: (i) normal stress differences, and (ii) yield criterion. The first was observed by Reynolds $(1885,1886)$ who called it dilatancy. The second peculiarity is that for a granular material to flow there is often a yield stress below which the particles do not undergo deformation. The yield condition is often related to the angle of repose, friction, and cohesion among other things. After the pioneering work of Bagnold $(1954,1956)$ many non-Newtonian models have been proposed. For a review of this aspect of the modeling activi- ties we refer the reader to the recent article by Elaskar and Godoy (1998).

Reiner $(1945,1948)$ proposed and derived a constitutive relation for wet sand whereby the concept of dilatancy is given a mathematical structure. This was accomplished through the relation between two tensors and the resulting second order effects in viscous and elastic media. This model does not take into account how the voidage (volume fraction) affects the stress. However, using this model, Reiner showed that application of a non-zero shear stress produces a change in volume. The constitutive relation of the type

$T_{i j}=F_{0} \delta_{i j}+2 \eta D_{i j}+4 \eta_{c} D_{i l} D_{l j}$

describing the rheological behavior of a non-linear fluid was named by Truesdell (see Truesdell and Noll, 1992) as the Reiner-Rivlin (see Rivlin, 1948) fluid, where in tensor notation the stress tensor $\mathbf{T}$ is related to $\mathbf{D}$, where $\mathbf{D}$ is the symmetric part of the velocity gradient (see Serrin, 1959 for a discussion of this model and the Stokesian fluid):

$\mathbf{T}=-p(\rho) \mathbf{I}+\alpha_{0} \mathbf{I}+\alpha_{1} \mathbf{D}+\alpha_{2} \mathbf{D}^{2}$

where $p$ is a function of the density and the $\alpha$ 's are function of $\rho, \operatorname{tr} \mathbf{D}$, and $\operatorname{tr} \mathbf{D}^{2}$. To incorporate the effects of volume fraction and its gradient Rajagopal and Massoudi (1990) and Rajagopal et al. (1994) derived a constitutive model that predicts the possibility of normal stress-differences and is also properly frame invariant (see also Cowin, 1974; Savage, 1979):

$\mathbf{T}=\left[\beta_{0}+\beta_{1} \nabla \phi \cdot \nabla \phi+\beta_{2} \operatorname{tr} \mathbf{D}\right] 1+\beta_{3} \mathbf{D}+\beta_{4} \nabla \phi \otimes \nabla \phi+\beta_{5} \mathbf{D}^{2}$

where

$\mathbf{D}=\frac{1}{2}\left[\nabla \mathbf{v}+(\nabla \mathbf{v})^{T}\right]$

Where 'tr' and $\nabla$ denote the trace and the gradient operators, respectively, ' $\because$ ' designates the inner product, ' $\otimes$ ' indicates the outer (dyadic) product of two vectors, $\rho=\rho_{s} \phi$, with $\rho_{s}$ being constant, $\phi(\mathbf{x}$, $t)$ is an independent kinematical variable called the volume distribution function and has the property $0 \leq \phi(\mathbf{x}, t)<\phi_{\max }<1$; and the $\beta$ 's are material properties, which in general are functions of the appropriate principal invariants of the density gradient and the symmetric part of the velocity gradient. They also assumed (Massoudi, 2001):

$\beta_{0}=k \phi ; \quad k<0$

$\left\{\begin{array}{l}\beta_{1}=\beta_{1}^{*}\left(1+\phi+\phi^{2}\right) \\ \beta_{2}=\beta_{2}^{*}\left(\phi+\phi^{2}\right) \\ \beta_{3}=\beta_{3}^{*}\left(\phi+\phi^{2}\right) \\ \beta_{4}=\beta_{4}^{*}\left(1+\phi+\phi^{2}\right) \\ \beta_{5}=\beta_{5}^{*}\left(\phi+\phi^{2}\right)\end{array}\right\}$

Such a quadratic dependence, at least for the viscosity $\beta_{3}$ is on the basis of dynamic simulations of particle interactions (Walton and Braun, 1986). For a detailed discussion of this model where specific interpretations are given to the coefficients and experimental techniques to measure these parameters are suggested (see Massoudi, 2004). For example, $\beta_{0}(\phi)$ is similar to pressure in a compressible fluid and is to be given by an equation of state, $\beta_{2}(\phi)$ is like the second coefficient of viscosity in a compressible fluid, $\beta_{1}(\phi)$ and $\beta_{4}(\phi)$ are the material parameters connected with the distribution of the granular materials, $\beta_{3}(\phi)$ is the viscosity of the granular materials, and $\beta_{5}(\phi)$ is similar to the viscosity term in the Reiner-Rivlin model often referred to as the cross-viscosity. This model has a similar structure to the Reiner-Rivlin model, whereby the effects of density (or volume fraction) gradients are also included. 
In this paper, we modify this model in such a way that the coefficient of viscosity can also depend on the shear rate. Specifically we make the additional assumptions:

$$
\begin{aligned}
& \beta_{0}=-p(\phi) \\
& \beta_{1}=\beta_{4}=0 \\
& \beta_{2}(\phi)=\beta_{20}\left(\phi+\phi^{2}\right) \\
& \beta_{3}(\phi, \operatorname{tr} \mathbf{D})=\beta_{30}\left(\phi+\phi^{2}\right) \Pi^{m / 2} \\
& \beta_{5}(\phi)=\beta_{50}\left(\phi+\phi^{2}\right)
\end{aligned}
$$

where

$$
\Pi=\frac{1}{2} \operatorname{tr}[2 \mathbf{D}]^{2}
$$

When $m<0$, the material is shear-thinning, and if $m>0$, it is shear-thickening. The justification for the dependence of the viscosities on the volume fraction, at least for the shear viscosity, stems from the numerical simulations of Walton and Braun (1986). The specific form of the pressure term is left open for now, but it should have a structure at least similar to $p=p_{\mathrm{f}}+p_{\mathrm{f}} \phi$ which can be re-written as $p=p_{\mathrm{f}}(1+\phi)$ indicating that as $\phi(\mathbf{x}, t)$ approaches zero or becomes zero, the stress reduces to the pure pressure of the fluid. Substituting Eq. (9) into Eq. (5), we obtain:

$$
\begin{aligned}
\mathbf{T}= & {\left[-p(\phi)+\beta_{20}\left(\phi+\phi^{2}\right) \operatorname{tr} \mathbf{D}\right] 1+\beta_{30}\left(\phi+\phi^{2}\right) \Pi^{m / 2} \mathbf{D} } \\
& +\beta_{50}\left(\phi+\phi^{2}\right) \mathbf{D}^{2}
\end{aligned}
$$

If for simplicity, we define

$$
\begin{aligned}
& \lambda=\beta_{20}\left(\phi+\phi^{2}\right) \\
& \mu=\beta_{30}\left(\phi+\phi^{2}\right) \Pi^{m / 2} \\
& \delta=\beta_{50}\left(\phi+\phi^{2}\right)
\end{aligned}
$$

Then,

$$
\mathbf{T}=[-p(\phi)+\lambda \operatorname{tr} \mathbf{D}] 1+\mu \mathbf{D}+\delta \mathbf{D}^{2}
$$

which has the same structure as a Reiner-Rivlin fluid where the material coefficients are now given by Eq. (12). This is the modified or generalized Reiner's model; in the next section we will use this equation to study the flow of wet sand (granules) between two flat plates.

It needs to be mentioned that among the many models that have been proposed for granular materials one can name the generalized Newtonian fluid (GNF) models, the visco-plastic (for example, the Bingham type) fluid models, the rate-dependent models (for example the hypo-plastic models), etc. (see Ancey, 2007; Gudehus, 1996; Kolymbas, 1991). Recently Jop et al. (2006) proposed a power-law model for granular materials where not only the viscosity depends on the shear rate, but also there is a yield criterion similar to the Drucker-Prager model (1952). The model presented in our paper as given by Eq. (11) or (13), also includes a shear rate dependent viscosity, but it differs from Jop et al. (2006) in the sense that: (1) the current model includes the term $\mathbf{D}^{2}$, thus it is a higher order model and in theory is capable of predicting at least one of the normal stress differences; and (2) all the material parameters depend on the volume fraction. Furthermore, in general, if we had used Eq. (5) as the basis of deriving a generalization of the Reiner's model, it would have been possible by giving a special structure to the material parameters, $\beta_{0}(\phi), \beta_{1}(\phi)$ and $\beta_{4}(\phi)$ to include the effects of yield stress via a Mohr-Coulomb type criterion but also the possibility of capturing both normal stress differences in a simple shear flow (see Massoudi and Mehrabadi, 2001 for details).

\section{Simple shear flow}

For a simple shear flow, i.e., flow between two horizontal plates a distance ' $h$ ' apart, with the lower plate fixed and the upper plate moving with a constant speed, the velocity field $\mathbf{v}$ and the volume function $\phi$ are assumed to be of the form

$$
\begin{aligned}
& \mathbf{v}=u(y) i \\
& \phi=\phi(y)
\end{aligned}
$$

It then follows that

$\mathbf{D}=\frac{1}{2}\left(\begin{array}{ccc}0 & u^{\prime} & 0 \\ u^{\prime} & 0 & 0 \\ 0 & 0 & 0\end{array}\right), \quad \mathbf{D}^{2}=\frac{1}{4}\left(\begin{array}{ccc}\left(u^{\prime}\right)^{2} & 0 & 0 \\ 0 & \left(u^{\prime}\right)^{2} & 0 \\ 0 & 0 & 0\end{array}\right)$

Also, notice that

$\operatorname{tr} \mathbf{D}=0, \quad \operatorname{tr}^{2}=\frac{1}{2}\left(\frac{d u}{d y}\right)^{2}, \quad$ and $\quad \Pi^{m / 2}=\left|\frac{d u}{d y}\right|^{m}$

Now, using Eqs. (14)-(16) in (13), we find that

$$
\begin{aligned}
& T_{x y}=\frac{1}{2}\left[\mu \frac{d u}{d y}\right] \\
& T_{x x}-T_{y y}=0 \\
& T_{y y}-T_{z z}=\left[\beta_{50}\left(\phi+\phi^{2}\right)\right]\left(\frac{d u}{d y}\right)^{2}
\end{aligned}
$$

If the term $\beta(\phi) \nabla \phi \otimes \nabla \phi$ were kept in the constitutive expression in Eq. (5), the model would be capable of exhibiting both of the normal stress differences. For the flowfield assumed by (14), Eq. (2), using (13), reduces to the three components in the $x, y$, and $z$ direction, respectively,

$$
\begin{aligned}
& \frac{1}{2} \frac{d}{d y}\left[\mu \frac{d u}{d y}\right]+\rho b_{x}=0 \\
& \frac{d}{d y}\left[-p(\phi)+\frac{\beta_{50}\left(\phi+\phi^{2}\right)}{4}\left(\frac{d u}{d y}\right)^{2}\right]+\rho b_{y}=0 \\
& \rho b_{z}=0
\end{aligned}
$$

where $b_{x}, b_{y}$, and $b_{z}$ are the components of the external body force, and $\mu$ is given by Eq. (12). Notice that with Eq. (14), the conservation of mass, Eq. (1) is automatically satisfied. Thus, we see that a motion of the form of Eq. (14) is only possible if the $z$-component of the body forces field is zero. Eqs. $(18 \mathrm{a}, \mathrm{b})$ form a system of two coupled second order non-linear ordinary differential equations, and in general have to be solved numerically. Appropriate boundary conditions are needed in order to have a well-posed problem. For the simple shearing motion assumed by (14), we have

$$
\begin{aligned}
& u(0)=0 \\
& u(h)=V
\end{aligned}
$$

where ' $h$ ' is the distance between the two plates. ${ }^{1}$ And for this special case, we only need one boundary condition for $\phi$,

$$
\phi(0)=\phi_{0}
$$

where $\phi_{0}$ is a constant. To obtain an analytical solution (closed form solution) to the above Eqs. (18a,b) under some idealized conditions, we furthermore assume

$b_{x}=b_{y}=b_{z}=0$

\footnotetext{
${ }^{1}$ It is possible that the no-slip condition may not be appropriate for all cases.
} 
Then, Eq. (18a) can be integrated once to give:

$\left[\mu \frac{d u}{d y}\right]=C_{1}$

where $C_{1}$ is a constant. Recalling that

$\Pi^{m / 2}=\left|\frac{d u}{d y}\right|^{m}$

we have

$\mu=\beta_{30}\left(\phi+\phi^{2}\right)\left|\frac{d u}{d y}\right|^{m}$

Substituting this in Eq. (22), we have

$\left[\beta_{30}\left(\phi+\phi^{2}\right)\left|\frac{d u}{d y}\right|^{m}\right] \frac{d u}{d y}=C_{1}$

Eq. (18b) can be also integrated once to give us

$-p(\phi)+\frac{\beta_{50}\left(\phi+\phi^{2}\right)}{4}\left(\frac{d u}{d y}\right)^{2}=C_{2}$

where $C_{2}$ is a constant. Now, it can be shown that the above Eqs. (25) and (26) admit a solution of the form

$$
\begin{aligned}
& u=\alpha y \\
& \phi=k
\end{aligned}
$$

where $\alpha$ and $k$ are constants. However, the system of Eqs. (18a,b) is non-linear and in general will admit additional solutions. This simple boundary value problem with all the basic assumptions specified should serve as a limiting case for more complicated flow geometries and flow conditions. Obviously, the effects of the interstitial fluid, slip at the wall, particle shape... are important issues which need to be studied.

\section{Concluding remarks}

In summary we have looked at the flow of a densely packed bed of particles (with liquid in the pores) between two horizontal flat plates. We have modified the constitutive relation originally derived by Reiner (1945), to describe dilatancy in wet sand, by suggesting that the shear viscosity would also depend on the shear rate and the volume fraction. As a result of this modification, the fluid referred to as the Reiner-Rivlin equation, now has the possibility of being shear-thickening (dilatant) not only due to shear rate, but also due to the dependence of the viscosity on the volume fraction. Finally, even though Reiner's model and its generalization in this paper are derived for wet sand, the effects of the moisture content, other than the pressure term which appears in the isotropic part of the stress tensor, are ignored [see Eq. (11)]. To include dynamical effects due to moisture or the presence of the fluid in the pores one needs to, in general, use a multi-component approach to fluidsaturated granular materials (see for example Wang and Hutter, 1999; Lagree and Lhuillier, 2006; Massoudi, 2010).

\section{References}

Ahmadi, G., 1980. On mechanics of saturated granular materials. Int. J. Non-linear Mech. Vol. 15, 251-262.

Ancey, C., 2007. Plasticity and geophysical flows: a review. J. Non-Newton. Fluid Mech. 142, 4-35.

Andrade, E.N.D.C., Fox, J.W., 1949. The mechanism of dilatancy. Proc. Phys. Soc. Lond. B62 (483).
Antony, S.J., Hoyle, W., Ding, Y., 2004. Granular Materials: Fundamentals and Applications. The Royal Society of Chemistry, Cambridge, UK.

Bagnold, R.A., 1954. Experiments on a gravity free dispersion of large solid spheres in a Newtonian fluid under shear. Proc. R. Soc. Lond. 225 (49)

Bagnold, R.A., 1956. The flow of cohesionless grains in fluids. Philos. Trans. R. Soc. Lond. A 249 (235).

Bolton, M.D., 1986. The strength and dilatancy of sand. Geotechnique 36, 65 .

Cowin, S.C., 1974. A theory for the flow of granular materials. Powder Technol. 9, 61-69.

Drucker, D.C., Prager, W., 1952. Soil mechanics and plastic analysis or limit design. Q. Appl. Math. 10, 157-165.

Duran, J., 2000. Sands, Powders, and Grains. Springer-Verlag, New York

Elaskar, S.A., Godoy, L.A., 1998. Constitutive relations for compressible granular materials using non-Newtonian fluid mechanics. Int. J. Mech. Sci. 40, 10011018.

Goldhirsch, I., 2003. Rapid granular flows. Annu. Rev. Fluid Mech. 35, 267-293.

Gudehus, G., 1996. A comprehensive constitutive equation for granular materials. Soils Found. 36, 1-12.

Hermann, H.J., 1999. Statistical models for granular materials. Physica A 263, 51-62.

Hermann, H.J., Luding, S., 1998. Modeling granular media on the computer. Continuum Mech. Thermodyn. Vol. 10, 189-231.

Jenkin, C.F., 1931. The pressure exerted by granular materials: an application of the principle of dilatancy. Proc. R. Soc. Lond. A 131, 53.

Jop, P., Forterre, Y., Pouliquen, O., 2006. A constitutive law for dense granular flows. Nature 441, 727-730.

Kolymbas, D., 1991. An outline of hypoplasticity. Arch. Appl. Mech. 61, 143-151.

Lagree, P.Y., Lhuillier, D., 2006. The Couette flow of dense and fluid-saturated granular media. Eur. J. Mech. B: Fluids 25, 960-970.

Massoudi, M., 2001. On the flow of granular materials with variable material properties. Int. J. Non-linear Mech. 36, 25-37.

Massoudi, M., 2004. Constitutive modelling of flowing granular materials: a continuum approach. In: Antony, S.J., Hoyle, W., Ding, Y. (Eds.), Granular Materials: Fundamentals and Applications. The Royal Society of Chemistry, Cambridge, UK, pp. 63-107.

Massoudi, M., 2010. A mixture theory formulation for hydraulic or pneumatic transport of solid particles. Int. J. Eng. Sci. 48, 1440-1461.

Massoudi, M., Boyle, E.J., 2001. A continuum-kinetic theory approach to the flow of granular materials: the effects of volume fraction gradient. Int. J. Non-linear Mech. 36, 637-648.

Massoudi, M., Mehrabadi, M.M., 2001. A continuum model for granular materials: considering dilatancy, and the Mohr-Coulomb criterion. Acta Mech. 152, 121-138.

Mehta, A. (Ed.), 1994. Granular Matter. Springer-Verlag, New York.

Rajagopal, K.R., Massoudi, M., 1990. A Method for Measuring Material Moduli of Granular Materials: Flow in an Orthogonal Rheometer. Topical Report, DOE/PETC/TR-90/3.

Rajagopal, K.R., Massoudi, M., Wineman, A.S., 1994. Flow of granular materials between rotating disks. Mech. Res. Commun. 21, 629-634.

Reiner, M., 1945. A mathematical theory of dilatancy. Am. J. Math. 67, 350362.

Reiner, Marcus, 1948. Elasticity beyond the elastic limit. Am. J. Math. 70, 433466.

Reiner, M., 1958. Rheology. In: Flugge, S. (Ed.), Handbuch Der Physik. SpringerVerlag, Berlin.

Reynolds, O., 1885. On the dilatancy of media composed of rigid particles in contact with experimental illustrations. Philos. Mag., Ser. 5 20, 469-481.

Reynolds, O., 1886. Experiments showing dilatancy, a property of granular material, possibly connected with gravitation. Proc. R. Inst. Gr. Brit. 11, 354-363.

Rivlin, R.S., 1948. The hydrodynamics of non-Newtonian fluids. Proc. R. Soc. Lond. 193, 260-281.

Rivlin, R.S., Ericksen, J.L., 1955. Stress deformation relations for isotropic materials. J. Rat. Mech. Anal. 4, 323-425.

Rowe, P.W., 1962. The stress-dilatancy relation far static equilibrium of an assembly of particles in contact. Proc. R. Soc. Lond. A 269, 500.

Savage, S.B., 1979. Gravity flow of cohesionless granular materials in chutes and channels. J. Fluid Mech. 92, 53-96.

Serrin, J., 1959. The derivation of stress-deformation relations for a Stokesian fluid. J. Math. Mech. 8, 459-469.

Tardos, G.I., 1997. A fluid mechanistic approach to slow, frictional flow of powders. Powder Technol. 92, 61-74.

Truesdell, C. (Ed.), 1965. The Rational Mechanics of Materials. Gordon and Breach Science Publishers, Inc., New York.

Truesdell, C., Noll, W., 1992. The Non-Linear Field Theories of Mechanics. SpringerVerlag, New York.

Walton, O.R., Braun, R.L., 1986. Stress calculations for assemblies of inelastic spheres in uniform shear. Acta Mech. 63, 73-86.

Wang, Y., Hutter, K., 1999. A continuum theory of fluid-saturated granular materials and its applications in gravitational flows. Rheol. Acta 38, 214-223. 